\title{
About Evaluation of Complex Dynamical Systems
}

\author{
Dmytro Polishchuk and Olexandr Polishchuk \\ Pidstryhach Institute for Applied Problems of Mechanics and Mathematics, National Academy of Sciences of Ukraine, \\ 3 baukova straße, Lviv 79000, Ukraine \\ Correspondence should be addressed to Olexandr Polishchuk; od_polishchuk@mail.ru
}

Received 30 April 2013; Accepted 17 September 2013

Academic Editor: Fuwen Yang

Copyright (C) 2013 D. Polishchuk and O. Polishchuk. This is an open access article distributed under the Creative Commons Attribution License, which permits unrestricted use, distribution, and reproduction in any medium, provided the original work is properly cited.

Methods are proposed for evaluation of complex dynamical systems, choice of their optimal operating modes, determination of optimal operating system out of given class of equivalent systems, system's timeline behaviour analysis on the basis of versatile multicriteria, and multilevel analysis of behaviour of system's elements.

\section{Introduction}

The study of different types of complex dynamical systems (technical, biological, social, economical) has attracted the attention of many researchers for a long time already [1-4]. Important direction of such a study is development of methods for evaluation of state, operating quality, and interaction between objects of those systems [5-10]. Among main problems arising in this case local and global evaluation of complex dynamical systems quality $[10,11]$ may be pointed out, as well as determination of their optimal operation modes [12], analysis of system behaviour during a certain period of time, and the choice of optimal operating system out of certain class of equivalent systems. We propose a unified approach to solving the above-listed problems. It is based on their comprehensive (which presupposes consideration of as many characteristics of system's elements as possible), multicriteria, and multiparameter analysis. In order to provide operative processing of results the developed evaluation is multilevel, which means formulation of conclusions of different generalization degrees: from locally determining the behaviour of particular characteristics of system's elements to finally determining quality of its operation in general. Problems considered in this paper and methods for their solution are illustrated with example of motion analysis of man's musculoskeletal system (MMSS) with prosthetic lower limb [13-15].

\section{Problems Formulation}

Let us consider dynamical system consisting of $N$ elements able to operate under $L$ modes. In order to simplify the statement, let us assume that behaviour of every element of system under $l$ th mode is described by set of characteristics $A_{n l, m}(t), m=\overline{1, M}, l=\overline{1, L}$, where $n$ is the component of characteristic corresponding to $n$th system's element, $n=$ $\overline{1, N}, t \in[0, T]$, where $T$ is the time for test research. Each of these characteristics results from experimental research or mathematical modelling for processes taking place in the system. To analyse the behaviour $A_{n, l, m}(t)$, let us use $K_{l}^{m}$ criteria. Let us denote with $\Omega_{n, l, m, k_{l}^{m}}$ and $\widetilde{\Omega}_{n, l, m, k_{l}^{m}}$ the domains of reference and permissible values for $A_{n, l, m}(t)$ characteristic according to $k_{l}^{m}, \Omega_{n, l, m, k_{l}^{m}} \subset \widetilde{\Omega}_{n, l, m, k_{l}^{m}}, k_{l}^{m}=$ $\overline{1, K_{l}^{m}}$, criterion. The term "equivalent" implies systems with the same content, type, and destination; the law of motion for which on the sequence of operating modes is described by set of characteristics $A_{n, l, m}(t), m=\overline{1, M}, l=\overline{1, L}, n=\overline{1, N}, t \in$ $[0, T]$, that are to satisfy defined set of criteria. Let us denote with $G_{Q}$ class of equivalent dynamical systems which consists of $Q$ elements.

While modelling MMSS with prosthetic lower limb as the multilink system of solid bodies, its motion may be described through rhythmical, kinematical, dynamical, and 
energy characteristics and so forth, [13-15]. Each of these characteristics represents $n$-dimensional vector function with its components describing peculiarities of system's elements behaviour (separate joints of human body and prosthesis applied) in the course of motion. Among the evaluation criteria the deviation from known average norm, walking asymmetry level, deviation from best practice running rehabilitation result, and so forth may be pointed out. The domain of reference values for characteristics components is represented by domain of their change in course of normal walking, 3\% level of walking asymmetry, known data on best prosthesis results, and so forth. Disabled persons of the same sex, age group, and health condition with same level of lower limb amputation and same or different types of prostheses applied make up the class of equivalent systems.

In this work we will consider following problems for complex systems evaluation.

(1) Evaluation of System's Element Operation Quality. Solution for this problem allows determining elements carrying potential threat of general system operation failures and analysing their impact on surrounding elements. For systems composed of elements of the same type, solution of this problem allows for determining elements operating in the best way, that is, reference elements. Finally, development of generalized conclusions on general system operating quality is based on results of system's elements evaluation.

(2) Choice of Optimal Mode for System Operation. Solution of this problem allows for determining most "comfortable" as well as potentially failure and extreme system operating modes.

(3) Evaluation of System Operation Quality. Solution of this problem allows for determining the general quality of system operation according to defined set of parameters, criteria, and operation modes.

(4) Choice of Optimally Operating System out of Given Class of Equivalent Systems. Solution of this problem allows us to determine the best (referential) or the worst systems of the class. Optimally functioning elements, modes, and systems determined in the evaluation process may be used along as practically reachable quality references.

(5) Analysis of System Operation History. Solution of this problem allows us to track and forecast the quality of system operation, determining trends of its development in the context of improvement or deterioration and to prevent possible failures in advance.

Turning to the issue of human prosthesis analysis, results of elements operation quality evaluation allow to define MMSS joints that are exposed to overload in the course of motion and distort its kinematics. Choice of optimal mode allows us to determine the most sparing pace of motion for disabled persons. Evaluation of system operation quality may therefore be interpreted as the quality of prosthesis for specific invalid. Choice of optimal system of the class defines the best running prosthetic result possible to be used along as evaluation criterion. If the class is represented by the set "disabled person-set of prostheses of different constructions," the choice of optimal system means determination of most favourable prosthesis construction for specific patient. Analysis of system behaviour during certain period of time allows for evaluating the process of invalid adaptation to prosthesis applied and so forth. Study of evaluation results with the presence of negative or close to negative conclusions provides strong reasons for improvement or change in rehabilitation methods applied.

\section{Parameters and Scales of Evaluation}

We will evaluate behaviour of the characteristic $A_{n, l, m}(t)$ under $k_{l}^{m}$ th criterion by means of parameters $h_{n, l}^{m, k, p}=$ $\left\|\alpha_{n, l}^{m, k}\right\|_{H_{p}[0, T]}$, where $\alpha_{n, l}^{m, k}(t)=\rho\left(A_{n, l, m}(t), \Omega_{n, l, m, k_{l}^{m}}\right)$ is a distance between $A_{n, l, m}(t)$ and domain of reference values for $n$th component of this characteristic under criterion $k_{l}^{m}$ and $H_{p}[0, T]$ is a line of functional spaces, for example, $C_{p-1}[0, T], W_{2}^{p-1}[0, T], p=\overline{1, P_{n, l}^{m, k}}$, or their combination, $k_{l}^{m}=\overline{1, K_{l}^{m}}, m=\overline{1, M}, l=\overline{1, L}, n=\overline{1, N}, t \in[0, T]$. Parameter values in uniform metric allow us to trace separate peaks or disturbance in behaviour of provided characteristic and its derivatives, and those ones in mean-squared metric allow us to define average value of their falling beyond domains of reference or permissible values limits.

Local evaluation under the $h_{n, l}^{m, k, p}$ parameter is performed as follows. Let us denote with $h_{n, l, m i n}^{m, k}$ its minimum and maximum permissible values accordingly. As usual, the value $h_{n, l, \min }^{m, k}=0$ corresponds to characteristic $A_{n, l, m}(t) \epsilon$ $\Omega_{n, l, m, k_{l}^{m}}$ and the value $h_{n, l, \max }^{m, k, p}=\max _{A_{n, l, m} \in \widetilde{\Omega}_{n, l, m, k_{l}^{m}}} h_{n, l}^{m, k, p}$. If continuous evaluation scale is accepted, evaluation of local behaviour for characteristic $A_{n, l, m}(t)$ under $k$ th criterion and $p$ th parameter is defined by means of formula

$$
e_{C, n, l}^{m, k, p}=\frac{v\left(h_{n, l, \max }^{m, k, p}-h_{n, l}^{m, k, p}\right)}{\left(h_{n, l, \max }^{m, k, p}-h_{n, l, \mathrm{~min}}^{m, k, p}\right)},
$$

where $v$ is the normalizing coefficient, for example, 10 . Then the highest positive evaluation will correspond to characteristic with values not falling beyond domain of reference values, zero evaluation corresponds to characteristic with values reaching the limits of domain of permissible values, and negative evaluation corresponds to characteristic with values falling beyond domain of permissible values limits. Extent of this falling beyond limits is determined by absolute value of $e_{C, n, l}^{m, k, p}$.

If discrete evaluation scale is accepted, every real value of functional $h_{n, l}^{m, k, p}$ within the limits of interval $\left[h_{n, l, m i n}^{m, k, p}, h_{n, l, m a x}^{m, k}\right]$ corresponds to integer number. Let us suppose that $I$ is the number of grades of integer scale, for example, 10 and $\delta_{i} \epsilon$ $[0,1], \delta_{i}<\delta_{i+1}, i=\overline{0(1) I}, \delta_{0}=0, \delta_{I+1}=1$. Then integer rating evaluation for characteristic $A_{n, l, m}(t)$ is defined by coefficient $e_{D, n, l}^{m, k, p}=i$ if $e_{C, n, l}^{m, k, p} / v \in\left[\delta_{i}, \delta_{i+1}[, i=\overline{0(1) I}\right.$. 
If the number of grades of integer scale is not great (2-5), its values correspond to those ones of conceptual evaluation scale, where every grade of discrete scale correspond to value "unsatisfactory," "satisfactory," "good," "excellent" in ascending order. It is obvious that under consecutive transition from continuous to conceptual scale becomes less distinctive. The latter one, as well as the discrete scale with low number of grades, is practically unacceptable to trace insignificant changes in system elements behaviour or forecasting of this behaviour. One more drawback of conceptual scale is the fact that grade "satisfactory" may denote any possible value from "almost good" to "slightly better than unsatisfactory". However it is convenient and comprehensible in cases of single or rare routine system evaluations.

The hybrid scale seems most convenient for practical use, which is more precise rating scale, which combines the benefits of continuous and conceptual scales. Let us develop it as follows. To evaluate characteristic $A_{n, l, m}(t)$ according to $k_{l}^{m}$ criterion by $n$th component we will use the pair of parameters $h_{n, l}^{m, k, C}=\left\|\alpha_{n, l}^{m, k}\right\|_{C_{0}[0, T]}$ and $h_{n, l}^{m, k, L}=\left\|\alpha_{n, l}^{m, k}\right\|_{L_{2}[0, T]}$, that denote their deviation from domain of reference values in uniform and mean-squared metrics. Let us consider the characteristic evaluation $e_{D, n, l}^{m, k, C}$ as "excellent" or equivalent to 5 if $h_{n, l}^{m, k, C}=0$ and "unsatisfactory" if $h_{n, l}^{m, k, C}>h_{n, l, m a x}^{m, k}$. Let us introduce such parameter $\delta \in(0,1)$ that integer rating evaluation $e_{D, n, l}^{m, k, C}$ according to parameter $h_{n, l}^{m, k, C}$ is "satisfactory" or equivalent to 3 if continuous evaluation $e_{C, n, l}^{m, k, C} / 2 \in(0, \delta)$ and "good" or equivalent to 4 if $e_{C, n, l}^{m, k, C} / 2 \in[\delta, 1)$. Construction precise rating evaluation is illustrated by a simple example. Let $\Omega_{n, l, m, k_{l}^{m}}=$ $a_{\text {ref }}=$ const, $t \in[0, T], \widetilde{\Omega}_{n l, m, m, k_{l}^{m}}=\left[a_{\mathrm{ref}}, a_{\max }\right] \times[0, T], a_{\max }=$ const, $\gamma=a_{\text {ref }}+\delta\left(a_{\max }-a_{\text {ref }}\right)$, and $a(t)=\alpha_{n, l}^{m, k}(t), t \in[0, T]$ (Figure 1). Then we will consider precise rating evaluations $e_{S, n, l}^{m, k, C}$ according to parameter $h_{n, l}^{m, k, C}$ that define presence and magnitude of disturbances in behaviour of characteristic $A_{n, l, m}(t)$ equivalent to

$$
\begin{aligned}
& -2, \text { if } e_{D, n, l}^{m, k, C}=2 ; \\
& -3+\left(a_{\max }-\|a(t)\|_{C_{0}[0, T]}\right) /\left(a_{\max }-\gamma\right), \text { if } e_{D, n, l}^{m, k, C}=3 ; \\
& -4+\left(\gamma-\|a(t)\|_{C_{0}[0, T]}\right) / \gamma, \text { if } e_{D, n, l}^{m, k, C}=4 ; \\
& -5, \text { if } e_{D, n, l}^{m, k, C}=5 .
\end{aligned}
$$

Then we will consider precise rating evaluations $e_{S, n, l}^{m, k, L}$ according to parameter $h_{n, l}^{m, k, L}$ that define mass character of disturbances in behaviour of characteristic $A_{n, l, m}(t)$ equivalent to

$$
\begin{aligned}
& -2, \text { if } e_{D, n, l}^{m, k, C}=2 ; \\
& -3+\left(\left(a_{\max }-\gamma\right) \sqrt{T}-\|a(t)-\gamma\|_{L_{2}[0, T]}\right) /\left(a_{\max }-\gamma\right) \sqrt{T}, \\
& \text { if } e_{D, n, l}^{m, k, C}=3 ; \\
& -4+\left(\|\gamma-a(t)\|_{L_{2}[0, T]} / \gamma \sqrt{T}, \text { if } e_{D, n, l}^{m, k, C}=4 ;\right. \\
& -5, \text { if } e_{D, n, l}^{m, k, C}=5 .
\end{aligned}
$$

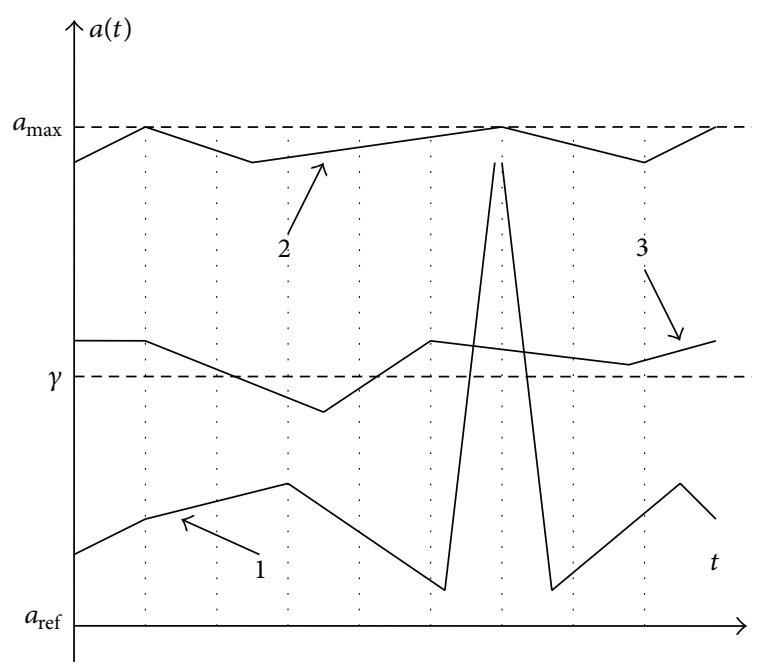

Figure 1

Then the pair of evaluations $e_{S, n, l}^{m, k, C}=3.05, e_{S, n, l}^{m, k}=3.98$ means the presence of low-number disturbances in behaviour of characteristic $A_{n, l, m}(t)$ (Figure 1, line 1). At the same time the pair of evaluations $e_{S, n, l}^{m, k, C}=3.01, e_{S, n, l}^{m, k, L}=3.02$ shows that quality of element operation considering characteristic studied and evaluation criterion is close to critical (Figure 1, line 2). Pair of evaluations $e_{S, n, l}^{m, k, C}=3.95, e_{S, n, l}^{m, k, L}=3.91$ shows that quality of element operation considering characteristic studied and evaluation criterion is close to "good" (Figure 1, line 3). That is, precise rating evaluations developed provide quite specific, reasoned, and understandable to average user information about the behaviour of evaluated system element. Evaluations $e_{S, n, l}^{m, k, C}, e_{S, n, l}^{m, k, L}$ are referred to as local and so on. In the same way they may be developed for the whole range of functional spaces $H_{p}[0, T], p=\overline{1, P_{n, l}^{m, k}}$, for arbitrary domains $\Omega_{n, l, m, k_{l}^{m}}$ and $\widetilde{\Omega}_{n, l, m, k_{l}^{m}}$.

In general the number of numerical values of local evaluations of element is evaluated with the number $S_{n}=$ $\sum_{l=1}^{L} \sum_{m=1}^{M} \sum_{k=1}^{K_{l}^{m}} P_{n, l}^{m, k}, n=\overline{1, N}$, and system elements selected for monitoring with the number $S=\sum_{n=1}^{N} S_{n}$. Let us take as an example motion of MMSS during walking [15]. When monitoring the behaviour of three pairs of MMSS joints (hip, knee, and ankle joints) while they are implementing 18 functions (walking in slow, normal, and fast speed along horizontal or inclined surfaces (up and down) with and without load), each of which is described by three characteristics (kinematical, dynamical, and energy) when evaluating according to 4 criteria (deviation from known normal areas of human walking [15], and level of motion asymmetry, deviation from best result reached, and level of motion stability [14]) according to 2 parameters (in uniform and mean-squared metrics), number of system elements evaluations $S_{n}=432, n=\overline{1,6}$ and general number of local evaluations $S=2592$. 


\section{Evaluation of System's Element Operation Quality}

It is obvious that direct analysis of totality of all numerical parameters of local evaluation is complex problem. For its solution the sequence of weighted averaged evaluations of different generalization level is developed on the basis of local evaluations set till the final conclusion regarding the operation quality of considered system's element is reached.

Such development is performed for every element according to the whole set of parameters, criteria, characteristics, and operating modes and presumes following levels of generalizations.

(1) According to the set of parameters for fixed criterion for evaluation of element's characteristic in operating mode specified,

$$
\begin{aligned}
& H_{n, l}^{m, k}=\frac{\left(\rho_{C} e_{S, n, l}^{m, k, C}+\rho_{L} e_{S, n, l}^{m, k, L}\right)}{\left(\rho_{C}+\rho_{L}\right)}, \\
& k=k_{n, l}^{m}=\overline{1, K_{n, l}^{m}}, \quad m=m_{n, l}=\overline{1, M_{n, l}}, \\
& l=\overline{1, L}, \quad n=\overline{1, N},
\end{aligned}
$$

where $\rho_{C}, \rho_{L}$ are weight coefficients defining evaluation parameters priority. Value received allows us to determine criteria under which evaluated element's characteristic in operating mode specified is unsatisfactory.

(2) According to the set of evaluation criteria for fixed element's characteristic in operating mode specified,

$$
\begin{gathered}
H_{n, l}^{m}=\frac{\left\langle\boldsymbol{\rho}^{C r}, \widetilde{\mathbf{H}}_{n, l}^{m}\right\rangle_{R^{K_{n, l}^{m}}}}{\left\langle\boldsymbol{\rho}^{C r}, \mathbf{1}\right\rangle_{R^{K_{n, l}^{m}}}}, \\
m=m_{n, l}=\overline{1, M_{n, l}}, \\
l=\overline{1, L}, \quad n=\overline{1, N},
\end{gathered}
$$

where $\langle\cdot, \cdot\rangle_{R^{K}}$ is a scalar product in Euclidean space $R^{K}, \mathbf{1}=\{1\}_{k=1}^{K}, \widetilde{\mathbf{H}}_{n, l}^{m}=\left\{H_{n, l}^{m, k}\right\}_{k=1}^{K_{n, l}^{m}}, \boldsymbol{\rho}^{C r}=\left\{\rho_{k}^{C r}\right\}_{k=1}^{K_{n, l}^{m}}$ is vector of weight coefficients defining evaluation criteria priority. Value received allows us to determine characteristics under which operation of evaluated element in operating mode specified is unsatisfactory.

(3) According to the set of characteristics in operating mode specified,

$$
\begin{gathered}
H_{n, l}=\frac{\left\langle\boldsymbol{\rho}^{C h}, \widetilde{\mathbf{H}}_{n, l}\right\rangle_{R^{M_{n, l}}}}{\left\langle\boldsymbol{\rho}^{C h}, \mathbf{1}\right\rangle_{R^{M_{n, l}}}}, \\
l=\overline{1, L}, \quad n=\overline{1, N},
\end{gathered}
$$

where $\widetilde{\mathbf{H}}_{n}^{m}=\left\{H_{n, l}^{m}\right\}_{m=1}^{M_{n, l}}, \boldsymbol{\rho}^{C h}=\left\{\rho_{m}^{C h}\right\}_{m=1}^{M_{n, l}}$ is vector of weight coefficients defining elements' characteristics priority. Value received allows us to determine operating modes in which operation of evaluated element is unsatisfactory.
(4) For element specified according to set of operating modes,

$$
H_{n}=\frac{\left\langle\boldsymbol{\rho}^{M o}, \widetilde{\mathbf{H}}_{n}\right\rangle_{R^{L}}}{\left\langle\boldsymbol{\rho}^{M o}, \mathbf{1}\right\rangle_{R^{L}}}, \quad n=\overline{1, N},
$$

where $\widetilde{\mathbf{H}}_{n}=\left\{H_{n, l}\right\}_{l=1}^{L}, \boldsymbol{\rho}^{M o}=\left\{\rho_{l}^{M o}\right\}_{l=1}^{L}$ is vector of weight coefficients defining operating modes priority. Value received allows us to determine system elements, operation of which is unsatisfactory. Improvement and modification of those elements allows us to increase general system operating quality. As for the problem of rehabilitation of disabled persons, unsatisfactory or close to unsatisfactory outcome of element evaluation means that selection of different design parameters for the prosthesis is required. Those parameters should help decrease load over retained joints of lower limbs and improve the kinematics of those persons' motion.

\section{Choice of Optimal Mode for System Operation}

If elements selected for monitoring possessed have similar sets of evaluation parameters and criteria, as well as characteristics, and operating modes, we are able to form generalized conclusions as to system's operation according to corresponding parameters, criteria, characteristics and operating modes. Let us develop the sequence of weighted average evaluations of different generalization degree on the basis of local evaluation set. This will allow us to analyse system's behaviour according to corresponding parameter, criterion, or characteristic while operating in specified mode.

(1) For separate evaluation parameter according to set of each characteristic's component for each evaluation criterion,

$$
\begin{gathered}
V_{l, C}^{m, k}=\frac{\left\langle\boldsymbol{\rho}^{E l}, e_{S, n, l}^{m, k, C}\right\rangle_{R^{N}}}{\left\langle\boldsymbol{\rho}^{E l}, \mathbf{1}\right\rangle_{R^{N}}}, \\
V_{l, L}^{m, k}=\frac{\left\langle\boldsymbol{\rho}^{E l}, e_{S, n, l}^{m, k, L}\right\rangle_{R^{N}}}{\left\langle\boldsymbol{\rho}^{E l}, \mathbf{1}\right\rangle_{R^{N}}}, \\
k=k_{n, l}^{m}=\overline{1, K_{n, l}^{m}}, \\
m=m_{n, l}=\overline{1, M_{n, l}}, \quad l=\overline{1, L},
\end{gathered}
$$

where $\boldsymbol{\rho}^{E l}=\left\{\rho_{n}^{E l}\right\}_{n=1}^{N}$ is vector of weight coefficient defining system's (elements') characteristic components priority. 
(2) For separate criterion according to set of evaluation parameters,

$$
\begin{gathered}
V_{l}^{m, k}=\frac{\left(\rho_{C} V_{l, C}^{m, k}+\rho_{L} V_{l, L}^{m, k}\right)}{\left(\rho_{C}+\rho_{L}\right)}, \\
k=k_{n, l}^{m}=\overline{1, K_{n, l}^{m}}, \\
m=m_{n, l}=\overline{1, M_{n, l}}, \quad l=\overline{1, L} .
\end{gathered}
$$

(3) For separate characteristics according to set of evaluation criteria,

$$
\begin{gathered}
V_{l}^{m}=\frac{\left\langle\boldsymbol{\rho}^{C r}, \widetilde{\mathbf{V}}_{l}^{m}\right\rangle_{R^{K_{n, l}^{m}}}}{\left\langle\boldsymbol{\rho}^{C r}, \mathbf{1}\right\rangle_{R^{K_{n, l}^{m}}}}, \\
\widetilde{\mathbf{V}}_{l}^{m}=\left\{V_{l}^{m, k}\right\}_{k=1}^{K_{n, l}^{m},} \\
m=m_{n, l}=\overline{1, M_{n, l}}, \quad l=\overline{1, L} .
\end{gathered}
$$

(4) For mode specified according to set of system elements characteristics,

$$
\begin{gathered}
V_{l}=\frac{\left\langle\boldsymbol{\rho}^{C h}, \widetilde{\mathbf{V}}_{l}\right\rangle_{R^{M_{n, l}}}}{\left\langle\boldsymbol{\rho}^{C h}, \mathbf{1}\right\rangle_{R^{M_{n, l}}}} \\
\widetilde{\mathbf{V}}_{l}=\left\{V_{l}^{m}\right\}_{m=1}^{M_{n, l}}, \quad l=\overline{1, L} .
\end{gathered}
$$

Evaluations $V_{l}$ allow us to determine operating modes in which system's operation is the worst. On the other hand, let us suppose that $K$ set of modes, $1 \leq K \leq l$, with the highest quality evaluation was received for system studied. Let us consider the procedure of optimal operating mode choice in case $K>1$.

Choice of optimal system operating mode is made in consideration of the following [16]. Let us presume that $\left\{a_{l}\right\}_{l=1}^{L}$ is optional set of real numbers under which $\sum_{l=1}^{L} a_{l}$. Among all numbers of this kind, value $\prod_{l=1}^{L} a_{l}$ reaches its maximal point when $a_{l}=A / L, l=\overline{1, L}$. If $\left\{a_{l}\right\}_{l=1}^{L}$ is the set of evaluations, it means that deviation of their values from simple average is minimal.

Let us suppose that we have a few modes with highest evaluations. We will consider mode (modes) for which maximum value of $\prod_{m=1}^{M_{n, l}} V_{l}^{m}$ is obtained optimal operating modes for dynamical system studied. As for the problem of rehabilitation of disabled persons it means that efforts between the joints are distributed more evenly in a given mode of motion or kinematics of motion similar to the motion of the normal human.

\section{Evaluation of System Operation Quality}

Using previous outcomes, evaluation of system operating quality may be received in two ways. Thus, the value

$$
H=\frac{\left\langle\boldsymbol{\rho}^{E l}, \widetilde{\mathbf{H}}\right\rangle_{R^{N}}}{\left\langle\boldsymbol{\rho}^{E l}, \mathbf{1}\right\rangle_{R^{N}}},
$$

where $\widetilde{\mathbf{H}}=\left\{H_{n}\right\}_{n=1}^{N}$ provides global system evaluation, that is, final conclusion regarding its operational quality.

The same evaluation will be received if the outcomes of system evaluation are generalized according to set of operating modes

$$
V=\frac{\left\langle\boldsymbol{\rho}^{M o}, \widetilde{\mathbf{V}}\right\rangle_{R^{L}}}{\left\langle\boldsymbol{\rho}^{M o}, \mathbf{1}\right\rangle_{R^{L}}},
$$

where $\widetilde{\mathbf{V}}=\left\{V_{l}\right\}_{l=1}^{L}$. It is obvious that $H \equiv V$.

\section{Choice of System with Optimal Operation}

Let us suppose that subclass $G_{\widetilde{Q}}, 1 \leq \widetilde{Q} \leq Q$, of systems with highest evaluations of operating quality was obtained from $G_{\mathrm{Q}}$ class. Let us consider the procedure of optimal system choice in case $1<\widetilde{Q}$, using algorithm applied for choice of optimal system operation mode. That is, we will consider the system (systems) of set $G_{\widetilde{Q}}$, for which maximum value of $\prod_{l=1}^{L} V_{l}$ is received, the class dynamical system with optimal operation. As for the problem of rehabilitation of disabled persons it means that transition from one to another mode of motion causes the minimal inconvenience for invalid.

\section{Analysis of Change in System Operation Quality}

The problem of analysis of change in systems operation quality in the timeline is similar to that one being considered in previous clause, the only difference being that class of equivalent systems is comprised of the same system but during different periods of time. Analysis for the best or the worst evaluation results will allow us to determine most or least favourable conditions for its operation. If the sequence (prehistory) of system evaluations $\left\{V\left(T_{j}\right)\right\}_{j=1}^{J}, J \geq$ 2 , received at point of time $T_{j}, j=\overline{1, J}$, is monotonically increasing, the quality of system operation increases, if it is monotonically decreasing, the quality decreases, and if it is close to constant value $\sum_{j=1}^{J} V\left(T_{j}\right) / J$, the quality is stable. Evaluations history allows us to perform at least short-term forecasting of system operation quality. Indeed, let us suppose that $\Phi(t)=\left\{\varphi_{j}(t)\right\}_{j=1}^{J}$ is the system of linearly independent functions, defined at the interval $\left[T_{1}, T_{J}\right]$. Let us develop function $V(t)=\langle\mathbf{A}, \boldsymbol{\Phi}(t)\rangle_{R^{J}}$, where $\mathbf{A}=\left\{a_{j}\right\}_{j=1}^{J}$ is the vector of unknown coefficients. Then forecasted value of system evaluation $V(t)$ at $T_{I+1}>T_{J}$ point of time is obtained from ratio $V\left(T_{J+1}\right)=\left\langle\mathbf{A}, \boldsymbol{\Phi}\left(T_{J+1}\right)\right\rangle_{R^{J}}$ where vector $\mathbf{A}$ is determined from condition $\left\langle\mathbf{A}, \boldsymbol{\Phi}\left(T_{k}\right)\right\rangle_{R^{J}}=V\left(T_{k}\right), k=\overline{1, J}$.

Prognostic analysis of precise evaluations allows to determine point of time when conceptual evaluation will be reduced by one unit. In particular, when the sequence $\left\{V\left(T_{j}\right)\right\}_{j=1}^{J}$ is monotonically decreasing, the time for next system study may be defined from condition $V(t) \geq V^{*}$ where $V^{*}$ is the value corresponding to conceptual evaluation decreased by one unit compared to that one determined at the moment of last examination. 


\section{Conclusions}

In this work unified approach is proposed for evaluation of complex systems operations on all levels of their structuring. It is obvious that substantiation of evaluation depends to a great extent on integrity and completeness of both corresponding modes sets as well as system elements characteristics and set of evaluation criteria and parameters, as well as adequacy of weighted coefficients defining their priority. The great number of local and generalized evaluations requires development of convenient ways for evaluation outcomes visualization and disaggregation of global conclusions of different levels for localisation of reasons for drawbacks discovered $[9,13,14]$.

Regarding the problems of disabled persons rehabilitation practices, methodology proposed allows, besides the above described, for solving the problems of comparative analysis for age, sex, and other peculiarities of human walking both in normal condition and with prosthetic lower limb, studying the impact of MMSS pathologies of different types on restriction of MMSS functional capabilities and impact of rehabilitation tools applied on reestablishment of such capabilities both in specific and in general cases, and performing comparative analysis of different rehabilitation methods and so on.

\section{References}

[1] A. L. Barabási, "The architecture of complexity," IEEE Control Systems Magazine, vol. 27, no. 4, pp. 33-42, 2007.

[2] S. Boccaletti, V. Latora, Y. Moreno, M. Chavez, and D. U. Hwang, "Complex networks: structure and dynamics," Physics Reports, vol. 424, no. 4-5, pp. 175-308, 2006.

[3] Y. Bar-Yam, "About engineering complex systems: multiscale analysis and evolutionary engineering," in Engineering SelfOrganising Systems: Methodologies and Applications, vol. 3464, pp. 16-31, Springer, London, UK, 2005.

[4] D. Hinrichsen and A. J. Pritchard, Mathematical Systems Theory, Springer, New York, NY, USA, 2005.

[5] J. Dombi, "Basic concepts for a theory of evaluation: the aggregative operator," European Journal of Operational Research, vol. 10, no. 3, pp. 282-293, 1982.

[6] A. Wittmuss, "Scalarizing multiobjective optimization problems," Mathematical Researches, vol. 27, pp. 255-258, 1985.

[7] C. L. Owen, "Evaluation of complex systems," Design Studies, vol. 28, no. 1, pp. 73-101, 2007.

[8] D. Roy and T. Dasgupta, "Evaluation of reliability of complex systems by means of a discretizing approach," International Journal of Quality \& Reliability Management, vol. 19, no. 6, pp. 792-801, 2002.

[9] L. Norros and P. Saviola, Usability Evaluation of Complex Systems, STUK, Helsinki, Finland, 2004.

[10] D. Polishchuk, O. Polishchuk, and M. Yadzhak, "Comparison of methods of complex system evaluation," Information Extraction and Processing, vol. 32, no. 108, pp. 110-118, 2010.

[11] A. D. Polishchuk, "Optimization of function quality of complex dynamical systems," Journal of Automation and Information Sciences, vol. 36, no. 4, pp. 39-44, 2004.

[12] O. Polishchuk, "Choice of optimal regimes for functioning of complex dynamical systems," Mathematical Methods and Physicomechanical Fields, vol. 48, no. 3, pp. 62-67, 2005.
[13] D. Polishchuk, O. Polishchuk, and M. Yadzhak, "Solution of some problems of evaluation of the complex systems," in Proceedings of the 15th International Conference on Automatic Control, pp. 968-976, Odesa, Ukraine, September 2008.

[14] O. Polishchuk, "Optimization of evaluation of man's musculosceletal system," in Computing Mathematics, vol. 2, pp. 360367, 2001.

[15] D. A. Winter, The Biomechanics and Motor Control of Human Gait: Normal, Elderly and Pathological, University of Waterloo Press, Waterloo, Canada, 1991.

[16] G. H. Hardy, J. E. Littlewood, and G. Pólya, Inequalities, Cambridge University Press, Cambridge, UK, 1988. 


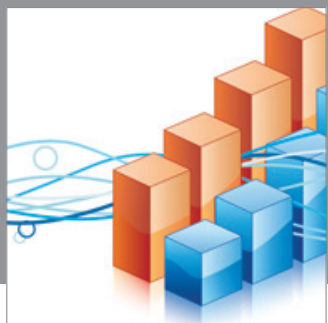

Advances in

Operations Research

mansans

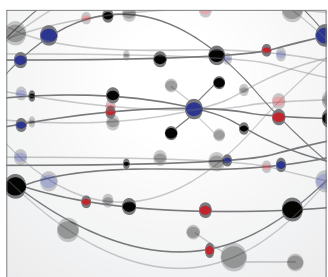

The Scientific World Journal
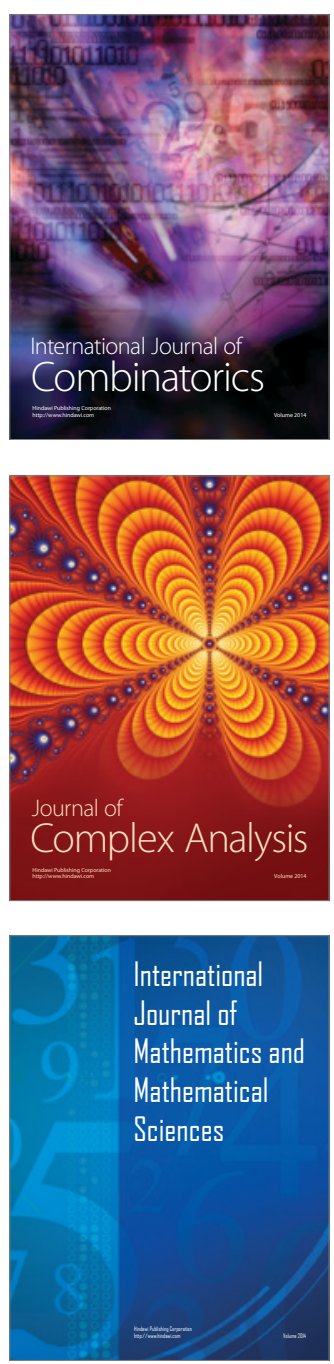
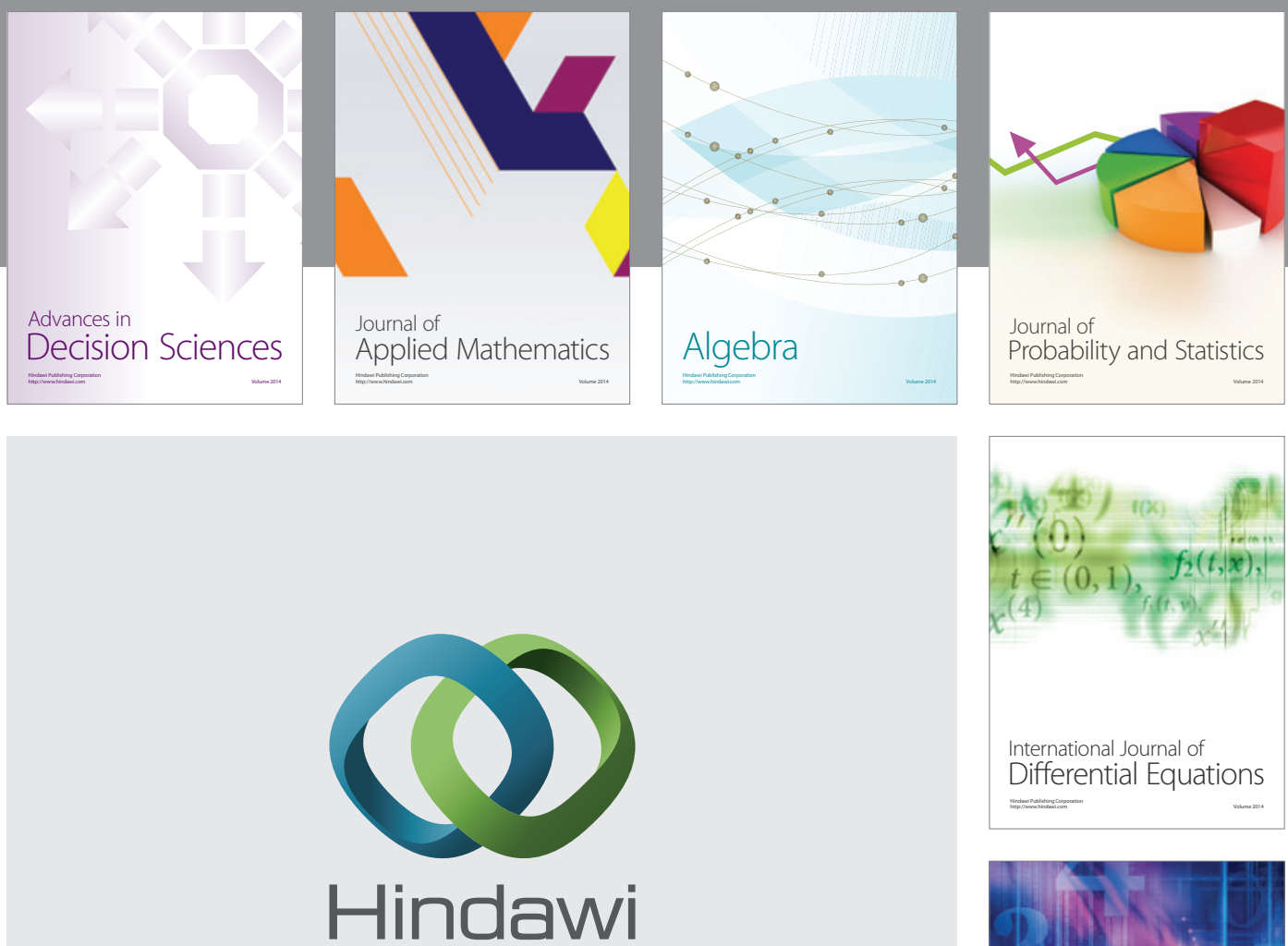

Submit your manuscripts at http://www.hindawi.com
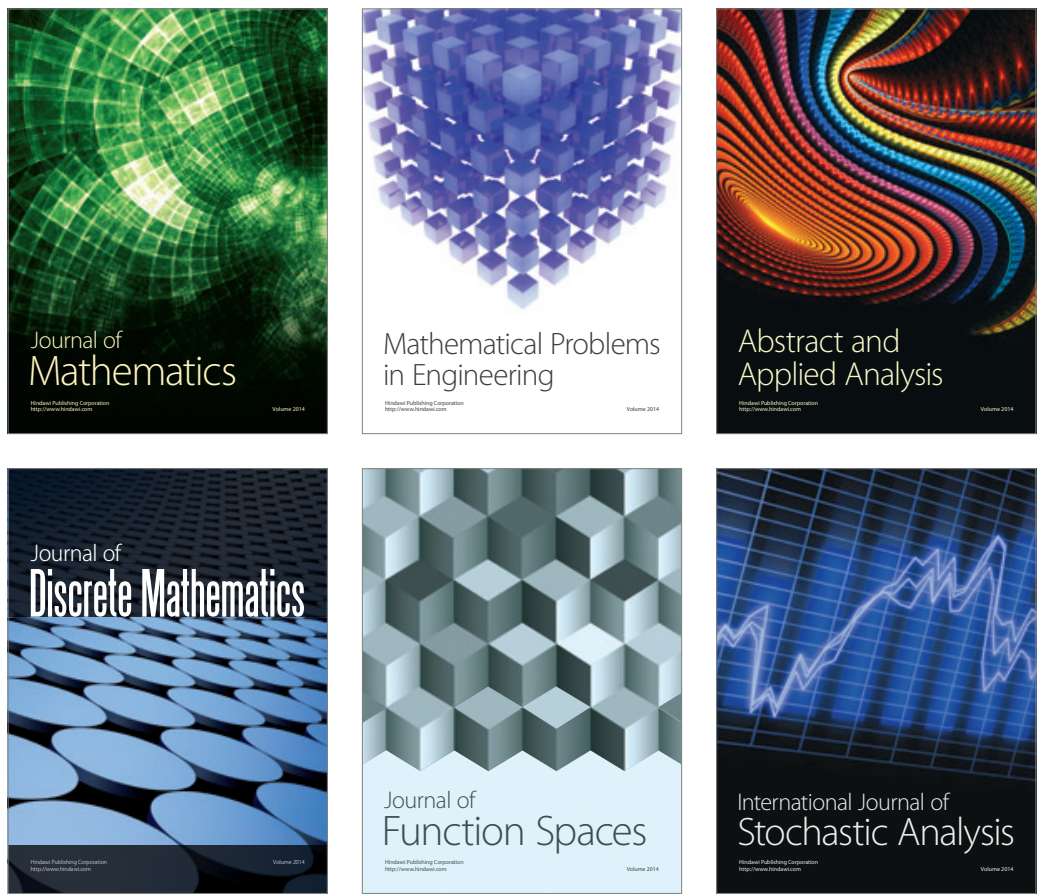

Journal of

Function Spaces

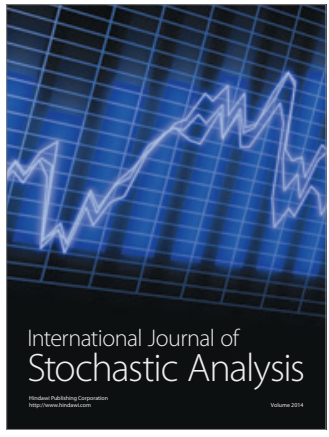

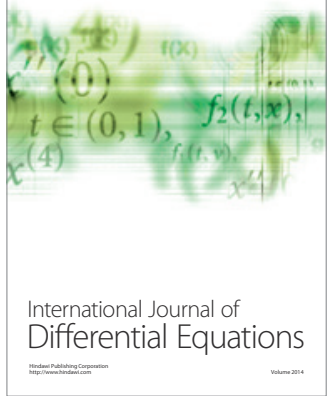
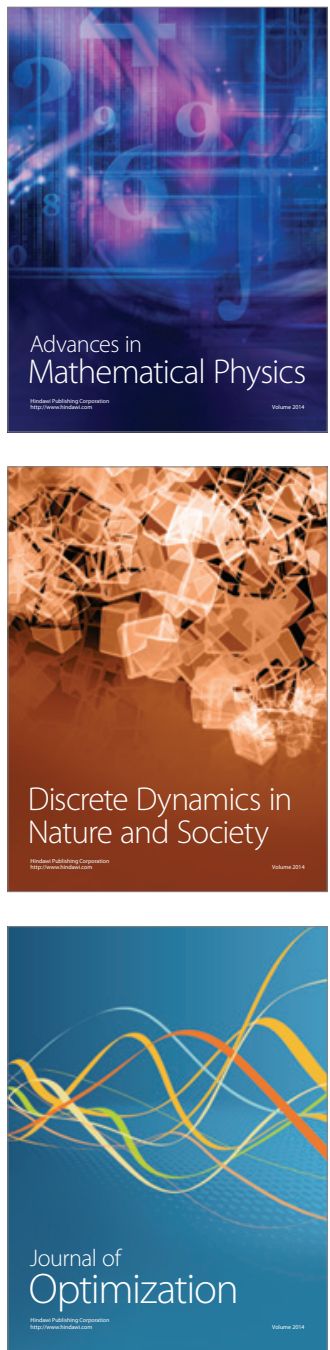